\title{
Spectrum of tablet computer use by medical students and residents at an academic medical center
}

Robert Robinson

Introduction: The value of tablet computer use in medical education is an area of considerable interest, with preliminary investigations showing that the majority of medical trainees feel that tablet computers added value to the curriculum. This study investigated potential differences in tablet computer use between medical students and resident physicians. Materials \& Methods: Data collection for this survey was accomplished with an anonymous online questionnaire shared with the medical students and residents at Southern Illinois University School of Medicine (SIU-SOM) in July and August of 2012. Results: There were 76 medical student responses (26\% response rate) and 66 resident/fellow responses to this survey ( $21 \%$ response rate). Residents/fellows were more likely to use tablet computers several times daily than medical students ( $32 \%$ vs $20 \%, p=$ $0.035)$. The most common reported uses were for accessing medical reference applications (46\%), e-Books (45\%), and board study (32\%). Residents were more likely than students to use a tablet computer to access an electronic medical record ( $41 \% \mathrm{vs}$ $21 \%, p=0.010)$, review radiology images ( $27 \%$ vs $12 \%, p=0.019)$, and enter patient care orders $(26 \%$ vs $3 \%, p<0.001)$. Discussion: This study shows a high prevalence and frequency of tablet computer use among physicians in training at this academic medical center. Most residents and students use tablet computers to access medical references, ebooks, and to study for board exams. Residents were more likely to use tablet computers to complete clinical tasks. Conclusions: Tablet computer use among medical students and resident physicians was common in this survey. All learners used tablet computers for point of care references and board study. Resident physicians were more likely to use tablet computers to access the EMR, enter patient care orders, and review radiology studies. This difference is likely due to the differing educational and professional demands placed on resident physicians. Further study is needed better understand how tablet computers and other mobile devices may assist in medical education and patient care. 
1 Spectrum of tablet computer use by medical students and residents at an academic medical center

\section{Author}

3 Robert Robinson, MD

4 Department of Internal Medicine

5 Southern Illinois University School of Medicine

6 Springfield, IL USA

7

8 


\section{${ }_{10}$ Introduction}

Tablet computers such as the Apple iPad and Kindle Fire are extraordinarily popular with the general public and physicians. These tablet computers generally have wireless networking capability and the ability to be customized by installing user selected "apps".

Apps are self-contained software applications with a diverse array of purposes ranging from entertainment to medical decision support. Over 1,600,000 apps are available for the Apple iOS platform (the operating system for the iPhone and iPad), with over 34,000 categorized as medical apps (PocketGamer, 2015). Medical apps typically cost less than \$5 (Robinson and Burk, 2012), and generally work on smartphones and tablet computers. The essential app for many physicians is their electronic medical record (EMR).

Ease of use and large screens make tablet computers a natural fit for EMR access, computerized physician order entry (CPOE), and radiology image review. As many as one-third of physicians in the United States use tablet computers in clinical settings, with $14-35 \%$ using these mobile devices to access an EMR (Robinson \& Burk, 2012; Sclafani, Tirrell \& Franko, 2013). Accessing an EMR via tablet computers can decrease the total time physicians spend logged into workstations while on duty (Hornq et al, 2012), improve the efficiency of inpatient medical care by facilitating earlier order entry (Patel et al, 2012), and appears to be preferred over traditional workstations in a hospital setting (Lehnbom et al, 2014). An observational study showed that tablet computers decrease inpatient data management time while increasing the time physicians spend interacting directly with patients (Fleishmann et al, 2015). These results are supported by systematic reviews that indicate tablet computer use can result in improved documentation, medical decision making, and physician efficiency

31 (Mickan et al, 2013; Mickan et al, 2014). In addition, patients embrace mobile technology and report 
tablet computer use by physicians as a positive aspect of their medical care (Strayer et al, 2010). These factors are likely to fuel even greater adoption of tablet computers by physicians.

The value of tablet computer use in medical education is an area of considerable interest, with some medical schools integrating tablet computers into their preclinical curriculum (Dolan, 2011). Preliminary investigations shows that the majority of students at a medical school felt that tablet computers were a positive addition that added value to the preclinical curriculum (George et al, 2013), and another medical school reports improved United States Medical Licensing Exam (USMLE) test scores after integration of tablet computers into the curriculum (Comstock, 2013). Studies of tablet computer use by medical trainees in the United States showed that point of care references (i.e. drug guides), board exam study resources, curricular materials, and EMR data were the most common types of medical resources used by medical students and residents on tablet computers during clinical rotations (Sclafani, Tirrell \& Franko, 2013; Robinson \& Burk, 2013; Nuss et al, 2014; Archibald et al, 45 2014). physicians at the same institution in hopes of providing insight into how these devices influence medical care and education. The working hypothesis was that tablet computer use and medical app use would substantially differ between medical students and resident physicians. 


\section{${ }_{51}$ Materials \& Methods}

52 Data collection for this survey was accomplished with an online questionnaire shared with the medical students and residents at Southern Illinois University School of Medicine (SIU-SOM) in July and August of 2012. The SIU-SOM is located in Springfield, Illinois and had an enrollment of 298 medical students and 314 residents and fellows at the time of this survey. Satellite training sites in Carbondale, Decatur, and Quincy, Illinois were also included. Training sites include 5 hospitals and many outpatient clinics in central and southern Illinois.

This anonymous survey was approved by the Springfield Committee for Research Involving Human Subjects (SCRIHS), the local institutional review board.

This survey asked respondents about tablet computer use, type of tablet computer (iPad, Android, other), medical app use, and frequency of medical app use. A scale for the frequency of medical app use was used. This scale of frequency included several times daily, daily, weekly, monthly, and never. Never was included because it was possible that students might only use tablet computers for personal purposes (email, games, etc.) and not use applications designed for medical purposes.

Qualitative variables were compared using Pearson chi $^{2}$ or Fisher's exact test and reported as frequency (\%), and $p$ values less than 0.05 were considered statistically significant. SPSS version 17.0 was used for data analysis.

An inactive copy of the survey instrument can be accessed at: http://goo.gl/wn5QU

SIU-SOM or any affiliated hospitals do not require or issue tablet computers to students or residents.

The training hospitals at SIU-SOM and the faculty outpatient practice have electronic medical records that are accessible via tablet computers in accordance with SIU-SOM and hospital policies. 


\section{Results}

74 There were 76 medical student responses (26\% response rate) and 66 resident/fellow responses to this

\section{${ }_{87}$ Discussion} 0.001). survey ( $21 \%$ response rate). Slightly over $50 \%$ of respondents used a tablet computer, with the Apple iPad being the most popular type of tablet computer used (Table 1). Tablet computer based use of medical apps one or more times daily was reported by $40 \%$ of respondents (Table 2 ). Residents/fellows were more likely to use tablet computers several times daily for medical apps than medical students ( $32 \%$ vs $20 \%, p=0.035$ ). A high percentage of medical students $(54 \%)$ and residents $(50 \%)$ report never using their tablet computers to access medical applications.

Respondents were asked about how they use tablet computers (Figure 1). The most common reported uses were for accessing medical reference applications, e-Books, and board study. Residents were more likely than students to use a tablet computer to access an electronic medical record ( $41 \%$ vs $21 \%, p=$ $0.010)$, review radiology images $(27 \%$ vs $12 \%, p=0.019)$, and enter patient care orders $(26 \%$ vs $3 \%, p<$

This study shows a high prevalence and frequency of tablet computer use, primarily in the form of iPads, among physicians in training at this academic medical center. Most residents and students use tablet computers to access medical references, e-books, and to study for board exams. The high frequency of e-book and other educational material use on tablet computers suggest that this may be an important avenue for medical educators to investigate. 
94 These results show a higher rate of resident tablet computer use (50\% vs. $13-19 \%)$ in clinical settings

95 and a higher rate of EMR access ( $41 \%$ vs. $14 \%$ ) than reported in a similar study investigating attending,

96 resident and fellow tablet computer use in other institutions (Sclafani J, Tirrell T and Franko O, 2013).

97 Rates of tablet computer use by medical students are similar to the results in a previously published

98 nationwide survey (Robinson and Burk, 2013). These differences in tablet computer utilization between

99 these studies may be related to information needs at different levels of training, the level of institutional

100 support for tablet computers, or other factors such as survey design.

101 Residents were more likely to use tablet computers for direct patient care such as accessing an EMR,

102 reviewing radiographs, and CPOE. This suggests that computers become integrated into the workflow of

103 residents at SIU-SOM, which is not unexpected given reports of improved resident efficiency and

104 increased time in direct patient care with tablet computer use (Hornq et al, 2012; Patel et al, 2012). This

105 also is likely a reflection of the transition of mobile computing needs from that of a student to that of a

106 practitioner.

107

Tablet computer use is likely complementary to high rates of smartphone use for many medical tasks by medical students and residents (Franko O and Tirrell T 2012; Payne K, Wharrad H and Watts K 2012).

110 Further investigation is needed to determine the preferred platform (phone vs. tablet) for access of

111 healthcare information.

113 One concern regarding the prevalence of a "bring your own" tablet computer for accessing healthcare

114 information is the relative ease in which information can be shared within a tablet computer. Text can 
115 be cut and pasted, images saved, and information shared through other applications. This is because

116 less than half of third year medical students think sharing patient photographs via social media is

117 "definitely a privacy concern" (Whipple E, Allgood K and Larue E, 2010) and 1\% of medical student and

118 resident Facebook profiles include identifiable photographs of patients in healthcare settings

119 (Thompson et al., 2011). Less than half of medical students password-protect patient data on their

120 mobile devices (Whipple E, Allgood K and Larue E, 2010). Medical schools must implement policies and

121 procedures sensitive to technological improvements that protect patient privacy in the new mobile

122 world.

123 As a single center study, the results of this survey may not be generalizable. Local factors, such as the

124 availability of EMR access via a tablet computer, are likely to have had significant effects on the patterns

125 of tablet computer use in this study. Additionally, this survey may have selection bias given the

126 voluntary nature of this survey. Despite these limitations, this survey shows widespread adoption and

127 daily clinical use of tablet computers in an academic medical center by medical students and residents.

129 Conclusions

130 Tablet computer use among medical students and resident physicians was common in this

131 survey. All learners used tablet computers for point of care references and board study. Resident

132 physicians were more likely to use tablet computers to access the EMR, enter patient care orders, and

133 review radiology studies. This difference is likely due to the differing educational and professional

134 demands placed on resident physicians. Further study is needed better understand when and how

135 tablet computers and other mobile devices may assist in medical education and patient care. 
${ }_{137}$ References

138 1. App store metrics. PocketGamer.Biz http://www.pocketgamer.biz/metrics/app-store/categories/

$139 \quad$ Accessed:5/19/2015

1402 2. Robinson R and Burk M. iHealthcare 2012: iPhone and iPad use by medical professionals.

141 Available at: http://itunes.apple.com/us/book/ihealthcare-2012/id507648390?mt=11

8. Mickan S, Tilson JK, Atherton H, Roberts NW, Heneghan C. Evidence of effectiveness of health care

7. Fleischmann R, Duhm J, Hupperts H, Brandt SA. Tablet computers with mobile electronic medical

6. Lehnbom EC, Adams K, Day RO, Westbrook Jl, Baysari MT. iPad use during ward rounds: an observational study. Stud Health Technol Inform. 2014;204:67-73.

records enhance clinical routine and promote bedside time: a controlled prospective crossover study. J Neurol. 2015 Mar;262(3):532-40. doi: 10.1007/s00415-014-7581-7. Epub 2014 Dec 5. professionals using handheld computers: a scoping review of systematic reviews. J Med Internet Res. 2013 Oct 28;15(10):e212. doi: 10.2196/jmir.2530. 
9. Mickan S, Atherton H, Roberts NW, Heneghan C, Tilson JK. Use of handheld computers in clinical practice: a systematic review. BMC Med Inform Decis Mak. 2014 Jul 6;14:56. doi: 10.1186/14726947-14-56.

10. Strayer SM, Semler MW, Kington ML, Tanabe KO. Patient attitudes toward physician use of tablet computers in the exam room. Fam Med. 2010 Oct;42(9):643-7.

11. Dolan, B. Nine medical schools that support mobile learning. 2011. Available at: http://mobihealthnews.com/12346/nine-medical-schools-that-support-mobile-learning/ Accessed: $5 / 19 / 2015$

12. George P, Dumenco L, Doyle R, Dollase R. Incorporating iPads into a preclinical curriculum: A pilot study. Med Teach. 2013 Feb 20. [Epub ahead of print]

13. Comstock, J. iPad-equipped medical school class scores 23 percent higher on exams. 2013. Available at: http://mobihealthnews.com/20311/ipad-equipped-medical-school-class-scores-23percent-higher-on-exams/ Accessed: 5/19/2015.

14. Robinson RL, Burk MS. Tablet computer use by medical students in the United States. J Med Syst. 2013 Aug;37(4):9959. doi: 10.1007/s10916-013-9959-y. Epub 2013 Jul 7.

15. Nuss MA, Hill JR, Cervero RM, Gaines JK, Middendorf BF. Real-time use of the iPad by third-year medical students for clinical decision support and learning: a mixed methods study. J Community Hosp Intern Med Perspect. 2014 Sep 29;4(4). doi: 10.3402/jchimp.v4.25184. eCollection 2014.

16. Archibald D, Macdonald CJ, Plante J, Hogue RJ, Fiallos J. Residents' and preceptors' perceptions of the use of the iPad for clinical teaching in a family medicine residency program. BMC Med Educ. 2014 Aug 20;14:174. doi: 10.1186/1472-6920-14-174.

17. Franko OI, Tirrell TF. Smartphone App Use Among Medical Providers in ACGME Training Programs. J Med Syst. 2012 Oct; 36(5):3135-9. DOI: 10.1007/s10916-011-9798-7. 
182
18. Payne KB, Wharrad H, Watts K. Smartphone and medical related App use among medical students and junior doctors in the United Kingdom (UK): a regional survey. BMC Med Inform Decis Mak. 2012 Oct 30;12:121. doi: 10.1186/1472-6947-12-121.

19. Whipple EC, Allgood KL, Larue EM. Third-year medical students' knowledge of privacy and security issues concerning mobile devices. Med Teach. 2012;34(8):e532-48. doi: 10.3109/0142159X.2012.670319. Epub 2012 Apr 10.

20. Thompson LA, Black E, Duff WP, Paradise Black N, Saliba H, Dawson K. Protected health information on social networking sites: ethical and legal considerations. J Med Internet Res. 2011 Jan 19;13(1):e8. doi: 10.2196/jmir.1590. 


\section{1}

Figure 1. Tablet computers use by stage of medical education

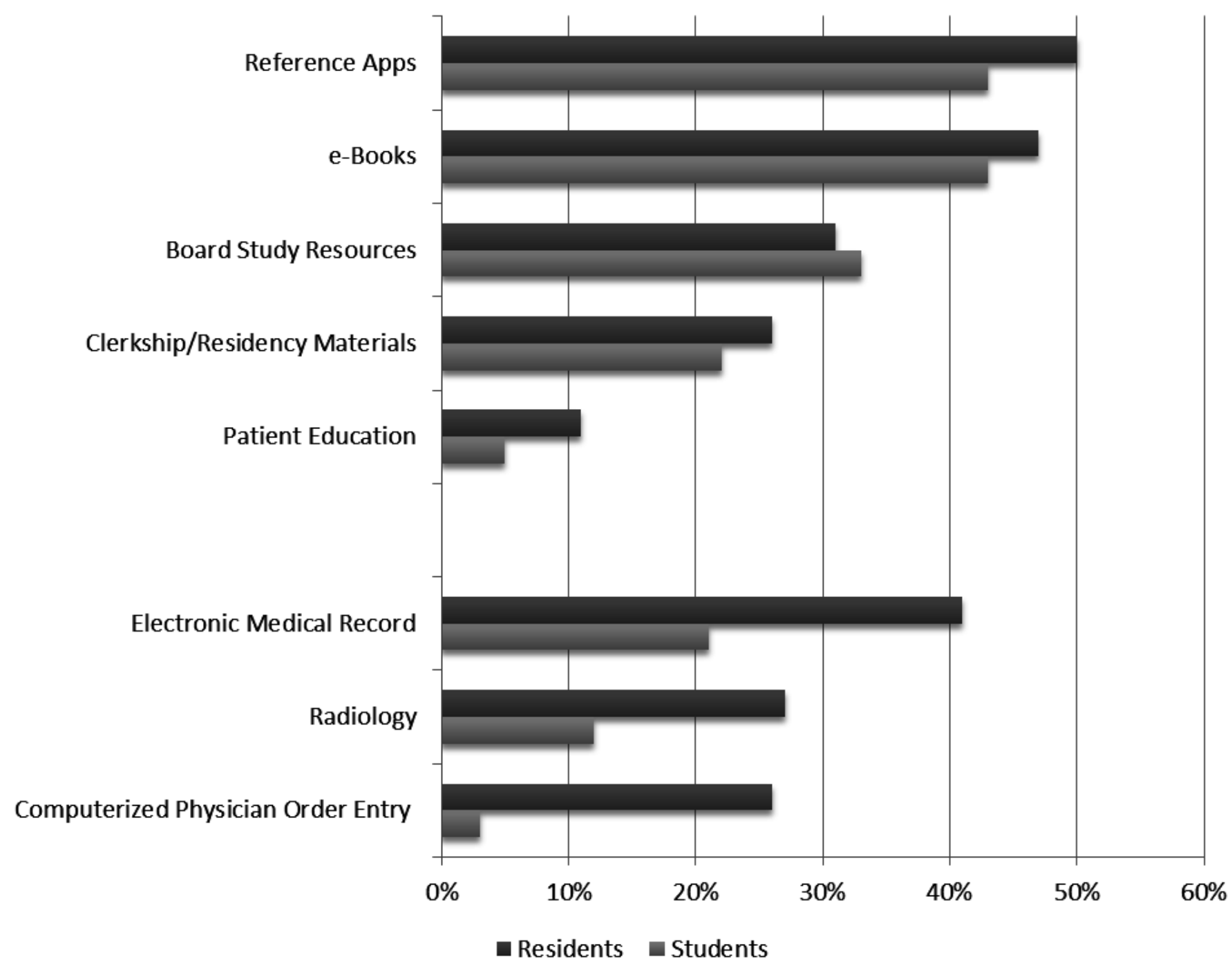




\section{Table $\mathbf{1}$ (on next page)}

Tablet computer use by physicians in training

Table 1. Tablet computer use by physicians in training 
Medical Students Residents/Fellows

\begin{tabular}{lll}
\hline Total respondents & 76 & 66
\end{tabular}

\begin{tabular}{ccc} 
Use a tablet computer & $39(51 \%)$ & $33(50 \%)$ \\
\hline Use an iPad & $35(46 \%)$ & $27(41 \%)$
\end{tabular}

\begin{tabular}{lllll} 
Use an Android Tablet & 4 & $(5 \%)$ & 6 & $(9 \%)$ \\
\hline
\end{tabular}

1

2

3

4 
Table 2 (on next page)

Frequency of medical app use by physicians in training

Table 2. Frequency of medical app use by physicians in training 


\begin{tabular}{|c|c|c|}
\hline & Medical Students & Residents/Fellows \\
\hline Total respondents & 76 & 66 \\
\hline Use medical apps & 35 (46\%) & $33(50 \%)$ \\
\hline Several times daily & $15(20 \%)$ & $21(32 \%)$ \\
\hline Daily & $10(13 \%)$ & $11(17 \%)$ \\
\hline Weekly & $10(13 \%)$ & $1(2 \%)$ \\
\hline Monthly & $0 \quad(0 \%)$ & $0 \quad(0 \%)$ \\
\hline Never & 41 (54\%) & 33 (50\%) \\
\hline
\end{tabular}

2 